\title{
Primary leiomyosarcoma of the pulmonary artery: Is aggressive treatment justified for a long survival?
}

\author{
Kalliopi Athanassiadi, MD, ${ }^{\text {a }}$ Christina Grothusen, MS, ${ }^{b}$ Michael Mengel, MD, ${ }^{c}$ and Axel Haverich, MD, ${ }^{a}$ \\ Hannover, Germany
}

$\mathrm{P}$ rimary malignant tumors of the pulmonary artery (PA) are rare with a dismal prognosis despite diagnostic, operative, and oncologic advances. ${ }^{1}$ We report 2 cases of primary leiomyosarcoma of PA with a long survival of 7 and 2.5 years including clinical presentation, diagnosis, and surgical treatment.

\section{Material}

Case 1. A 61-year-old woman was admitted to our department with suspected chronic pulmonary thromboembolism resulting in a complete occlusion of the right PA. ${ }^{2}$ The patient had chest pain followed by a progressive limitation of physical fitness and dyspnea (New York Heart Association classes II and III). Computed tomography (CT) scan revealed a hypodense formation with complete occlusion of the right PA. A lung perfusion-scintigraphy confirmed an almost complete loss of perfusion of the right lung, and a cardiac catheterization showed a mild pulmonary hypertension. Preoperative biopsy revealed sarcoma. After staging with cerebral magnetic resonance imaging and abdomen CT scan, the patient underwent a median sternotomy to right pneumonectomy and partial resection of the right main PA wall under cardiopulmonary bypass and reconstruction with a pericardial patch. The final histologic diagnosis stated leiomyosarcoma of low differentiation (G3) of PA with invasion of the proximal PA resection area (Figures 1 and 2). Subsequently, the patient underwent a second operation under extracorporeal circulation with complete resection of the PA trunk including the left PA. The reconstruction was performed with a $6-\mathrm{cm}$ polytetrafluoroethylene (Gore-Tex, Flagstaff, Ariz) prosthesis. The postoperative recovery was uneventful. In a follow-up of 7 years, she is alive with no recurrence. Her quality of life and exercise tolerance have improved.

Case 2. A 65-year-old woman was admitted to our hospital because of progressing dyspnea (New York Heart Association classes III and IV) and chest pain. Her medical history was not remarkable. Perfusion scintigraphy revealed absence of perfusion

From the Departments of Thoracic and Cardiovascular Surgery, ${ }^{\mathrm{a}}$ Cardiology and Angiology, ${ }^{\mathrm{b}}$ and Pathology, ${ }^{\mathrm{c}}$ Medical School of Hannover, Hannover, Germany.

Received for publication Jan 14, 2006; accepted for publication Feb 7, 2006.

Address for reprints: Kalliopi Athanassiadi, MD, Department of Thoracic and Cardiovascular Surgery, Medical School of Hannover, Carl-Neuberg Str. 1, 30627 Hannover, Germany (E-mail: kallatha@otenet.gr).

J Thorac Cardiovasc Surg 2006;132:435-6

0022-5223/\$32.00

Copyright $\odot 2006$ by The American Association for Thoracic Surgery doi:10.1016/j.jtcvs.2006.02.057

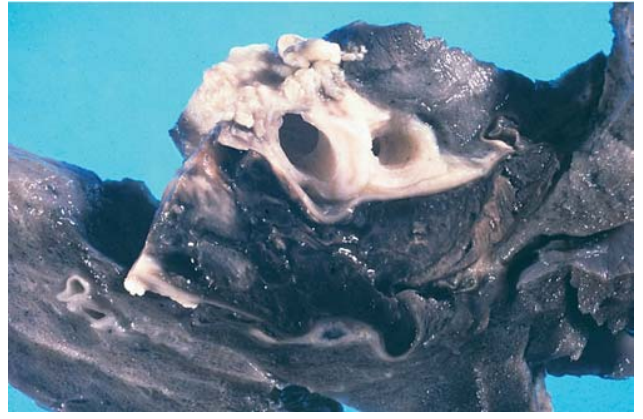

Figure 1. Cross section of pulmonary hilus with intraluminal tumor growth.

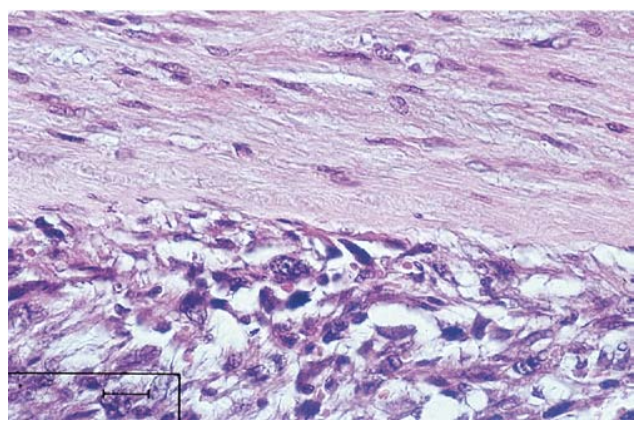

Figure 2. Histologic section of PA.

of the left lung, and CT scan showed a lobulated mass in the left PA outside the pericardium. No metastases were found, and the patient underwent a left posterolateral thoracotomy to intrapericardial pneumonectomy. The intraoperative diagnosis stated a primary leiomyosarcoma of low differentiation (G3) with free resection margins. The postoperative course was complicated with respiratory insufficiency caused by pneumonia. Preventive radiotherapy was suggested, but the patient refused. In a follow-up of 2.5 years she is still alive with no recurrence.

\section{Discussion}

Leiomyosarcomas of the PA are rare mesenchymal tumors with a poor prognosis and a mean survival of 1.5 months if no treatment is administered. ${ }^{1,2}$ Only 39 cases have been reported in the literature. Patients have a mean age of 50 years and show an equal distribution of gender. ${ }^{3}$ Its intraluminal growth is usual, leading to a progressive obstruction of right ventricular blood flow into the 
pulmonary circulation and a varying obstruction of pulmonary perfusion, thus resulting in clinically unspecific symptoms of dyspnea, cough, or impaired right ventricular function mimicking pulmonary thromboembolism in the majority of cases. ${ }^{2-4}$

The radiologic diagnosis can still be difficult despite the technologic advances in imaging techniques. ${ }^{1,5}$ Preoperative biopsy is the only way to establish a proper diagnosis, and it is essential to accurately plan the surgical approach. ${ }^{5}$

The only treatment of choice is radical surgical resection. ${ }^{4}$ Depending on tumor localization and distal extension, surgical treatment includes PA resection, a polytetrafluoroethylene (Gore-Tex) homograft, or even xenograft reconstruction and pneumonectomy. The use of cardiopulmonary bypass may be essential in patients in whom the tumor originates from the PA trunk or the pulmonary valve. As a palliative treatment, endovascular stent grafting may be an alternative therapeutic option in inoperable cases. Furthermore, neoadjuvant or adjuvant chemotherapy has been mentioned in the literature with doubtful results.

Prolonged tumor-free survival is rare. A 10-month survival is reported in the literature for different sarcomas of $\mathrm{PA},{ }^{5}$ whereas in our series survival was 7 and 2.5 years, respectively.

\section{Conclusion}

Early diagnosis along with aggressive and extensive surgical treatment in patients with PA leiomyosarcoma without any extrathoracic metastases may provide an opportunity for prolonged survival in addition to palliation.

\section{References}

1. Dumont P, Diot P, Aupart MR, Toumieux B. Leiomyosarcoma of the pulmonary artery. Ann Thorac Surg. 1998;66:2089-91.

2. Mengel M, Fraund S, Haverich A, Kreipe HH. Primary leiomyosarcoma of the pulmonary artery - a case report. Pneumologie. 2000; 54:20-3.

3. Mazzucco A, Luciani GB, Bertolini P, Faggian G, Morando G, Ghimenton C. Primary leiomyosarcoma of the pulmonary artery: diagnostic and surgical implications. Ann Thorac Surg. 1994;57:222-5.

4. Choong CK, Lawton JS, Moon MR, Damiano RJ Jr. Failure of medical therapy for pulmonary "thromboembolic" disease: beware the unsuspected primary sarcoma of the pulmonary artery. $J$ Thorac Cardiovasc Surg. 2004;128:763-5.

5. Hoffmeier A, Semik M, Fallenberg EM, Scheld HH. Leiomyosarcoma of the pulmonary artery-a diagnostic chameleon. Eur J Cardiothorac Surg. 2001;20:1049-51.

\title{
A new approach to the assessment of aortic cusp geometry
}

\author{
Hans-Joachim Schäfers, MD, PhD, Benjamin Bierbach, MD, and Diana Aicher, MD, Homburg/Saar, Germany
}

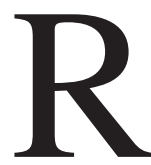

epair of the aortic valve is a new surgical approach to the treatment of aortic regurgitation. Regurgitation of the aortic valve can be caused by cusp distortion, root dilatation, or a combination of both. For good functional reconstruction, all pathologic components present have to be addressed. Good results have been achieved with valve-preserving aortic replacement, ${ }^{1}$ but isolated aortic valve reconstruction (ie, cusp repair for prolapse) still remains a challenge to surgical judgment.

The main problem in repair of aortic cusps is assessment of pathology and the repair result. Root dilatation can easily be quantified preoperatively by means of echocardiography or intraoperative measurements. Cusp geometry, however, is difficult to quantify by means of echocardiography. Measurements of cusp

From the Department of Thoracic and Cardiovascular Surgery, University Hospitals of Saarland, Homburg/Saar, Germany.

Received for publication March 29, 2006; accepted for publication April 5, 2006.

Address for reprints: Hans-Joachim Schäfers, MD, PhD, Department of Thoracic and Cardiovascular Surgery, University Hospitals of Saarland, 66421 Homburg/Saar, Germany (E-mail: h-j.schaefers@uniklinikum-saarland.de).

J Thorac Cardiovasc Surg 2006;132:436-8

$0022-5223 / \$ 32.00$

Copyright $\odot 2006$ by The American Association for Thoracic Surgery doi:10.1016/j.jtcvs.2006.04.032 dimensions are difficult to standardize intraoperatively, and these dimensions determine cusp configuration only in conjunction with sinus dimensions. ${ }^{2}$

The typical configuration of a normal aortic valve is not only characterized by root dimensions but also the configuration of the cusps. This includes a typical height difference between the central free margins and the aortic insertion lines (Figure 1, A). ${ }^{3}$ We have designed a caliper that allows easy and reproducible measurement of this height difference, which we call effective height as opposed to 2-dimensional cusp height. ${ }^{2}$ This measurement allows for identification of prolapse in the native cusps and assessment of prolapse correction after valve repair.

\section{Technical Description}

After cardioplegia, stay sutures are placed in the aortic commissures and kept under tension in the exact direction of the commissures (as seen from the center of the root). The caliper is placed such that the longer end rests on the lowest (ie, central) point of the insertion line. The shorter end is pushed to the free margin, with the curve accommodating the margin (Figure 1,B). The height difference of the cusp (free edge to insertion) can be measured in millimeters.

In normal aortic valves we have found effective height to range from 8 to 10 millimeters, which correlated $\pm 1 \mathrm{~mm}$ with intraoperative transesophageal measurements. In approximately 50 repair operations, we have found good correlation between effective 